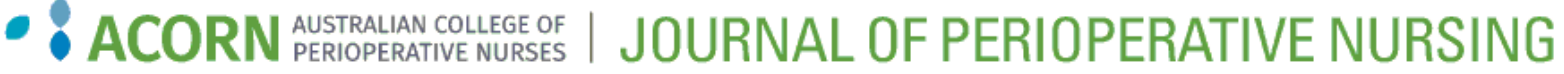

\title{
The effectiveness and compliance of surgical face mask wearing in the operating suite environment: An integrated review
}

Follow this and additional works at: https://www.journal.acorn.org.au/jpn

(c) (i)

This work is licensed under a Creative Commons Attribution 4.0 License.

\section{Recommended Citation}

Ogo, Noriko and Foran, Paula (2020) "The effectiveness and compliance of surgical face mask wearing in the operating suite environment: An integrated review," Journal of Perioperative Nursing: Vol. 33 : Iss. 4 , Article 2.

Available at: https://doi.org/10.26550/2209-1092.1105

https://www.journal.acorn.org.au/jpn/vol33/iss4/2

This Article is brought to you for free and open access by Journal of Perioperative Nursing. It has been accepted for inclusion in Journal of Perioperative Nursing by an authorized editor of Journal of Perioperative Nursing. 


\section{Authors}

Noriko Ogo

MCN (Perioperative Nursing),

BN, RN, MACORN

Dr Paula Foran

PhD, RN, FACORN, FACPAN, MACN

\title{
The effectiveness and compliance of surgical face mask wearing in the operating suite environment: An integrated review
}

\begin{abstract}
Problem identification: Surgical face masks have been used for over a century in the operating room (OR); firstly, as it has been believed that they may reduce surgical site infections (SSIs) in patients and, secondly, as personal protective equipment (PPE) for staff. However, recent studies have reported disparities in the efficacy of wearing surgical face masks including inconsistency in their use. This integrative review aims to investigate the effects of surgical masks in the OR, in regard to maintaining both patient and staff safety, and will discuss compliance or lack thereof in face mask usage for the entire multidisciplinary team.

Literature search: Four electronic health databases were used to identify the relevant research: CINAHL, Medline, EMCare and Cochrane Library. The reference lists of retrieved articles were also manually searched and appropriate literature retrieved.
\end{abstract}

Data evaluation synthesis: Inclusion and exclusion criteria were used revealing that 15 articles fully met the criteria which were critiqued (see Table 2 in Supplemental material for the literature matrix).

Implications for practice: SSIs are multifactorial and no reviewed studies looked at causative considerations such as comorbidities and other variables (such as hypothermia) which could all contribute to this post-operative complication. However, there is evidence to suggest that surgical face masks are useful in decreasing the biological load in the theatre environment. They are also a vital piece of PPE that should be worn to protect the perioperative team from blood and bodily fluid splatter that commonly occur.

Keywords: surgical mask, surgical site infection, PPE, operation theatre, staff compliance

\section{Introduction}

Traditionally there are two reasons for staff to wear surgical masks in the OR: firstly, to protect surgical sites from microorganisms transferred from the faces and respiratory tract of scrubbed staff and, secondly, to protect health care professionals from sprays and splashes of patients' blood and body fluids during surgery? Surgical site infections (SSIs) contribute to the increase in the burden of both patients and health care systems by increasing the length of hospitalisation, the cost of care, mortality and morbidity². Since surgical masks were introduced as a preventive practice for SSIs, over a hundred years ago, they have been used to provide a physical barrier between the mouth and nose of health care personnel and surgical wounds of patients ${ }^{3}$.

\section{Problem identification}

Despite the long-standing use of surgical face masks to prevent wound infection, a Cochrane systematic 
review conducted by Da Zhou, Sivathondan and $\mathrm{Handa}^{4}$ in 2015 concluded there was no statistically significant difference in the risk of SSIs between two cohorts, one masked and one non-masked. In the following year a second Cochrane systematic review by Vincent and Edwards was published which investigated the same question; however, their research only included 'clean' surgical procedures ${ }^{5}$. The cohort $(n=653)$ included patients who underwent 'clean' surgery where the respiratory, alimentary, genital or urinary tracts, that may contain bacteria, were not surgically treated ${ }^{5}$. Armed with the knowledge that there may be a lower risk of SSIs when conducting 'clean' surgery, it was hypothesised that the formation of a SSI in this cohort would make it more reasonable to attribute an infection to other factors such as a higher airborne bacterial load from the use or non-use of surgical face masks. However, results of this research were inconclusive, and it was unclear whether the use of a surgical face mask impacted the occurrence of SSIs in clean surgery 5 .

Standards for Perioperative Nursing in Australia (the ACORN Standards) states that surgical masks should be worn in operating theatres during procedures, when proximal to open sterile or aseptic clinical supplies and when there is a risk of blood or body fluid splash ${ }^{6}$. Although there are detailed criteria about the use of surgical masks in the ACORN Standards (Figure 1), ACORN acknowledges that using a surgical mask remains controversial in some health care settings and conflicting evidence may exist ${ }^{6}$. ACORN further suggests that individual health care settings should make their decision on best available evidence and clinician's judgment ${ }^{6}$.
Personnel have a duty to:

- wear disposable, high filtration masks:

- whenever a sterile/aseptic field is being prepared or maintained

- in the restricted areas during surgical procedures

- whenever there is risk of blood or body fluid splash

- wear masks that meet the Australian standards

- select the appropriate mask for the surgical procedure or infectious nature of the patient, and

- apply the mask securely to completely cover the nose and mouth

- secure the mask according to manufacturer's instructions and avoid touching the mask once it is applied

- remove the mask completely when it is no longer required, touching only the tapes/elastic, and discard into the appropriate receptacle

- perform hand hygiene following mask disposal

- avoid hanging masks around the neck when not in use.

Figure 1: Criteria 6.1-6.3 from ACORN standard 'Perioperative attire'

This integrative review aims to investigate the effects of wearing surgical masks in the $\mathrm{OR}$, and related compliance and behaviours of the multidisciplinary perioperative team.

\section{Literature search}

\section{Design}

This review follows an integrative approach which enables a broad review of the available literature. It includes various study methodologies to provide a comprehensive understanding of particular problems in health care settings ${ }^{7,8}$. It incorporates a fivestage process comprising problem identification, literature search, data evaluation and synthesis, data integration, and results presentation and discussion?.

\section{Literature search methods}

To identify research on the effect of wearing surgical masks in the perioperative environment and the compliance and behaviour of theatre staff, electronic health database searches of the literature were conducted using CINAHL, Medline, EMCare and Cochrane Library. The reference lists of retrieved articles were also manually examined to identify additional research papers relevant to the topic.

The initial search was limited to studies published between 2015 and 2020 and written in English. One salient paper from 2013 was subsequently retrieved.

\section{Inclusion and exclusion criteria}

Inclusion criteria included peer reviewed articles that reported the effects of the use of a surgical mask in the OR, those that investigated the efficacy of wearing surgical masks, and those that investigated the compliance and behaviour of operating theatre staff towards wearing surgical masks.

Exclusion criteria included studies that focused on a surgical helmet system, theatre traffic and respiratory infections. The terms used to conduct the search in the databases were based on the PICO (population, intervention, comparison, outcome) acronym, and Boolean operators 'OR' and 'AND' were used to combine synonyms and concepts of the PICO respectively. Terms were surgical mask OR surgical facemask AND 


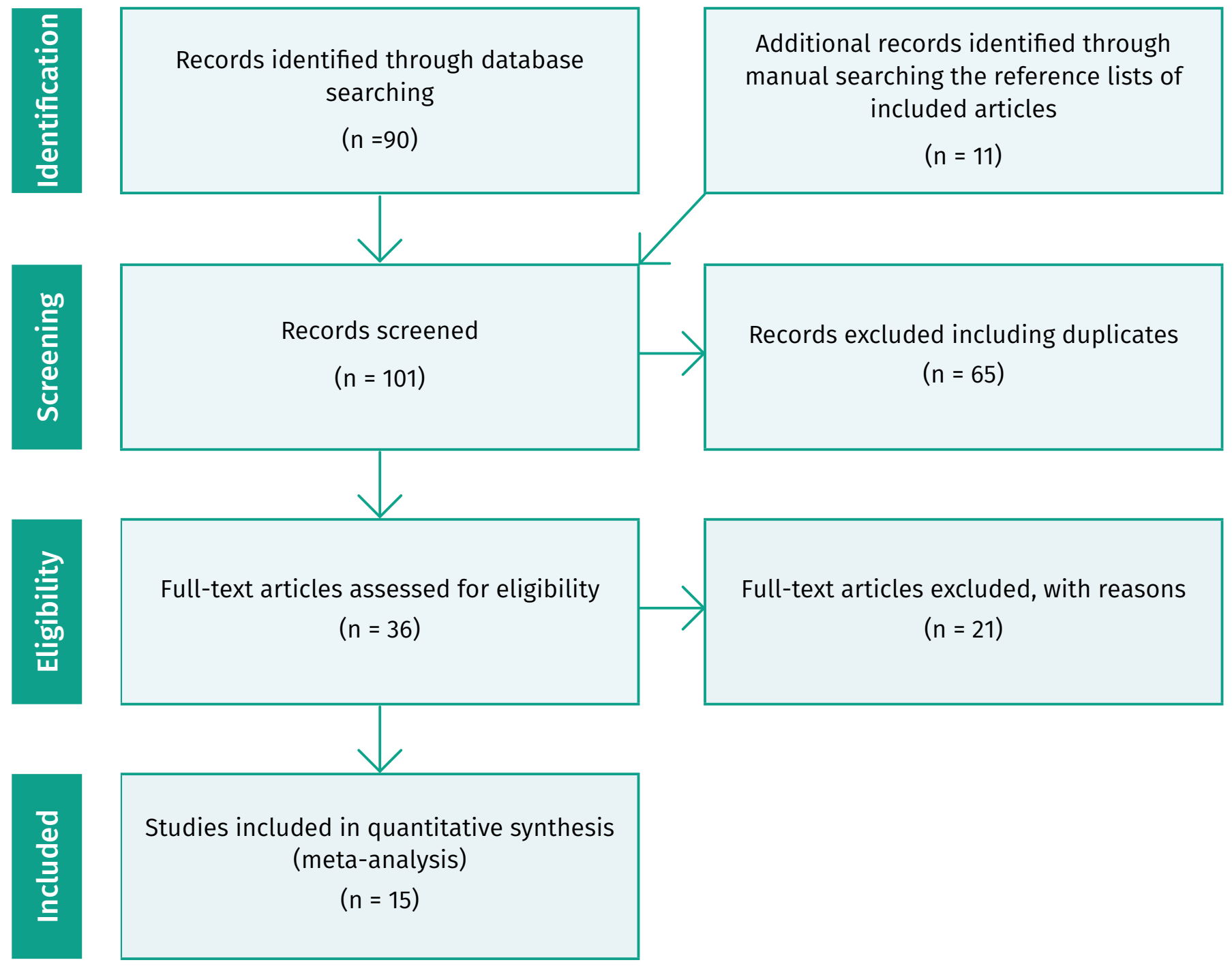

Figure 2: PRISMA flow diagram of search strategy

surgical site infection OR surgical wound contamination, with the article title, abstracts and body.

Articles that met the study inclusion criteria were summarised within the PRISMA (Preferred reporting items for systematic reviews and meta-analyses) flowchart (Figure 2) ${ }^{9}$. Ninety articles were initially retrieved from the electronic health database search and additional articles were added by manual searching of the reference lists and subsequent citations of the retrieved articles. After removing duplicates and papers that failed the inclusion criteria and critiquing process, 15 articles remained for this review.

\section{Data evaluation and synthesis}

\section{Data evaluation}

The studies discussed in this review employed different methodologies including systematic review, randomised controlled trial (RCT), comparative study with concurrent control, observational study, retrospective cohort study and literature review. The retrieved articles were appraised using the relevant critical appraisal skills programme (CASP) checklists ${ }^{10,11}$. RCTs provide the strongest evidence for a single study with minimal bias ${ }^{8}$. However, only one RCT was found as it is considered unethical, due to personal risk, for surgical staff to be randomly controlled to wear or not wear surgical masks during procedures.

A literature matrix was used to organise and critique the chosen literature and is attached as supplemental material. 


\section{Data extraction}

The extracted data included the authors, year of publication, country of research, study aims, study design, level of evidence using National Health and Medical Research Council (NHMRC) hierarchy of evidence, results and conclusions ${ }^{12}$. Limitations of each study were identified and considered in relation to the validity of the quality and findings of the studies. All the extracted data and limitations were summarised and demonstrated in the literature matrix to help the author prepare themes and critique and compare the outcomes from each article.

\section{Data analysis}

A narrative approach was used to synthesise the data of the included articles in order to compare, contrast, critically evaluate and interpret the findings. The extracted data revealed nine themes which are presented in the discussion. These themes were:

- reduction in aerosol colonyforming units (CFU) in the OR

- surgical masks and facial hair

- types of surgical mask and efficacy

- staff behaviour regarding overall face and hair coverings

- compliance with Centre for Disease Control and Prevention (CDC) guidelines

- negative impact on the relationship with patients

- speech intelligibility

- personal protective equipment (PPE)

- limitations of the efficacy of surgical face masks.

\section{Results presentation and discussion}

Reduction in aerosol colony-
forming units (CFU) in the OR

A recent experimental study by Howard et al. investigated the use of different face masks including a full facepiece powered air-purifying respirator (PAPR), a full facepiece PAPR with a shoulder-length hood, a surgical mask, and no facial covering (as a positive control to determine contamination-reduction effectiveness) $)^{13}$. Findings revealed a statistically significant difference $(p<0.0115)$ with the use of surgical masks decreasing aerosolised droplet contamination by 98.48 per cent compared with wearing no facial covering $^{13}$. While a small sample size, this research correlated with the results from a cohort study by Stockwell et al. who reported the amount of aerosol CFU counts was significantly decreased when wearing any type of surgical mask compared with no surgical masks during a cough test $(p<0.001, n=35)^{14}$. These findings reveal a decrease in CFU or biological load from wearing a surgical face mask in the OR.

\section{Surgical masks and facial hair}

Facial hair has always been a contentious issue in infection prevention. Perry et al. conducted research to investigate whether clinicians' facial hair may contribute to increased bacterial shedding and subsequent SSIs ${ }^{15}$. The results showed a statistically significant difference with bacterial shedding from bearded men being lower in the face mask cohort than those without surgical masks $(p<0.02)^{15}$. Statistical significance was greater in participants with beards two centimetres or longer in length $(p<0.03)$ although there was no statistical evidence that a bearded clinician increased the risk of SSIs by bacterial shedding while wearing a surgical face mask $(p<0.9)^{15}$. Interestingly, the addition of wearing a non-sterile surgical balaclava hood was shown not to be statistically significant in further reducing the amount of bacterial shedding $(p<0.6)^{15}$. This indicates that a surgical face mask may provide as much protection in reducing biological load as a non-sterile surgical balaclava hood, especially among clinicians with beards.

\section{Types of surgical mask and efficacy}

Patel et al. conducted an in vitro study on cough etiquette using two manikins under various conditions in simulated rooms to assess the contributing factors of wearing a surgical face mask to exposure reduction during coughing and tidal breathing $(n=22)^{16}$. They used three types of surgical masks: an N95 class filtering respirator, a natural fit earloop surgical mask and a SecureFit Ultra ${ }^{\text {TM }}$ fitted surgical mask ${ }^{16}$. The differences noted between mask types were significant indicating that the major mechanism of protection was the level of filtration, patterns of airflow in a particular setting and the ability of the mask to fit the face ${ }^{16}$. The most superior mask was the fitted N95 respirator with a petroleum jelly seal, as fit testing is complex in manikins ${ }^{16}$. Tidal breathing revealed that source control was comparable to or better than mask or respirator protection on the receiver, suggesting that surgical masks may be an important additional defence in preventing the spread of respiratory infections ${ }^{16}$. 


\section{Staff behaviour regarding overall face and hair coverings}

An observational study by Loison et al.17 assessed compliance with wearing surgical attire and traffic flow during surgery $(n=1615)$. Compliance with wearing surgical attire was observed for eight criteria, two of which related to wearing masks. Full compliance was seen in 56 per cent of personnel ${ }^{17}$. The position of a surgical mask (placed incorrectly over the nose and mouth) was the third highest contributor to non-compliance after presence of hand jewelry and position of head cover. Although not statistically significant, it is interesting to note that nursing staff showed the highest compliance (99\%) followed by surgeons (95\%) and anaesthetists $(89 \%)^{17}$. These results were similar to an observational study by Dallolio et al. 18 in which data of staff behaviours was collected $(n=10)$ showing that 65 per cent of theatre staff wore caps and masks correctly, having completely contained their hair and beard and covering mouth and nose. Scrubbed staff (surgeons and instrument nurses) showed the highest compliance (89.5\%) followed by unscrubbed staff (68.7\%) and personnel who weren't directly related to the procedure such as anaesthetists or nurses who came to ask for information $(47.4 \%)^{18}$.

\section{Compliance with Centre} for Disease Control and Prevention (CDC) guidelines

Herron et al.19 conducted an observational study to evaluate theatre staff compliance with manufacturer and CDC guidelines for using surgical masks ( $n=$ 1034). Only 18 per cent of staff demonstrated full compliance with the CDC guideline when applying a surgical mask, with compliance being higher in 'clean' procedures such as orthopaedic surgery and lower in surgeries classified as 'unclean'. Orthopaedic surgery reported the highest compliance $(73 \%)^{19}$. These results corresponded with the result from the study conducted by Louison et al. which reported that orthopaedic surgery showed statistically significant findings of highest compliance compared with other specialities $(p<0.01)^{17}$.

The different trends among the surgical specialities in adhering to guidelines may be influenced by the fact that the majority of SSIs are caused by a patient's endogenous flora and acquired in the operating theatre during surgery when microorganisms can reach the open surgical site ${ }^{19}$. Thus, the surgeons who perform ‘unclean' surgery are more likely to consider their aerosolised breath is insignificant during procedures, resulting in the low compliance with using a surgical mask $^{19}$. Additionally, an observational descriptive study conducted by Kang et al. investigated the participants' behaviours regarding PPE use ${ }^{20}$. Their findings noted staff having doubts about the effectiveness of PPE, including masks, caused by a lack of knowledge or awareness of the scientific evidence and this may be a barrier to compliance with using $\mathrm{PPE}^{20}$.

\section{Negative impact on the relationship with patients}

To understand the views of surgeons about wearing a surgical mask in operating theatres, Ahmad et al. ${ }^{21}$ conducted a survey among plastic surgeons. The quality of the study method and questionnaire were low level and not consistent; thus, many limitations were identified. For example it was unclear how many participants, divided into two groups, completed the survey; how the questionnaire was sent to participants; the countries where the plastic surgeons actually practiced; the association between the use of masks and the types of procedures from the questions; and the consent or ethical considerations were not mentioned $^{21}$. However, this study was conducted from multiple health services worldwide and revealed some interesting reasons for surgeons' low compliance with wearing a surgical mask. The majority of participants reported they did not wear a surgical mask when patients were entering the theatre to ease the patients' anxiety. Surgeons wearing spectacles also reported they found it difficult to cope with the fog effect while breathing in the mask especially when the nose is covered ${ }^{21}$.

Further results of an RCT by Wong et al. ${ }^{22}$ supported one of the findings from Ahmad et al. They reported wearing face masks during consultations negatively impacted on the patient's perception of the clinician's empathy $(p<0.04)$ although there was no statistically significant difference in patient enablement $(p<0.87)$ and satisfaction $(p<0.25)$ between the group in which clinicians wore masks and the non-mask group ${ }^{22}$. This study was conducted in a primary care clinic 22 ; thus the patients' expectations of health care professionals may have been different to patients' expectations in an operating theatre where surgical masks may be more acceptable. However, this illustrated that removing surgical masks for patients entering the theatres may minimise the negative impact on the quality of relationship with the patients.

\section{Speech intelligibility}

When investigating how wearing a surgical mask impacts on the ability of staff to communicate within the health care environment, Palmiero et al. reported on speech intelligibility, 
and the perceived quality of sound transmission ${ }^{23}$. The two types of masks which are typically used in the OR are protective face masks and N95 respirators. Results showed that compared to a no-mask condition both types of masks had some impact on speech intelligibility. Protective face masks had lower impact (3\% and $4 \%$ deviation for the two models tested) than N95 respirators (13\% and $17 \%$ deviation for the two models tested) ${ }^{23}$. The findings tell us that surgical masks covering the mouth and nose can decrease speech intelligibility and the wearer's ability to verbally communicate in the operating theatre, which is commonly a very loud environment ${ }^{23}$.

This study indicated that wearing surgical masks may interfere with communication between the perioperative team or between theatre staff and patients which are surrounded by many types of noises ${ }^{23}$.

\section{Personal protective equipment}

Cook et al. conducted an experimental study during spinal surgery $(n=46)$ and reported debris was highest on the surgeons' face shield (83\%) followed by the first assistant (35\%) and scrubbed technician/nurse $(0 \%)^{24}$. Moreover, debris was identified more in procedures where aggressive instruments were used such as a burr, osteotome or an interbody cage. The research by Cook et al. was limited to spinal surgery ${ }^{24}$; however, it has been shown that all types of surgery have some degree of risk of splash associated with them, and laparoscopic surgery shows a higher risk of splash particularly when the pneumoperitoneum is decompressed ${ }^{19}$. Therefore, scrubbed staff, especially surgeons, would benefit from using a surgical mask during surgery as part of normal PPE.
Furthermore, benefits of wearing a surgical mask for unscrubbed staff were studied in a systematic review by Spruce ${ }^{25}$. In this review, Spruce describes an observational, descriptive and non-experimental study involving 8500 surgical procedures conducted by White and Lynch reporting that blood was detected on the heads and necks of 26 per cent of scrubbed staff and 17 per cent of unscrubbed staff. This highlights that wearing surgical masks can also protect unscrubbed personnel from potential exposure to splash from the surgical field ${ }^{25}$. Thus, surgical masks should also be used as effective PPE by both scrubbed and unscrubbed theatre staff members in operating theatre for their own infection prevention.

\section{Limitations of the efficacy of surgical masks}

In order to maximise the benefits of wearing surgical masks, several factors should be noted in addition to wearing a surgical mask correctly. Zhiqing et al. concluded that a surgical mask could be a potential source of SSI when it is used for extended hours, especially over two hours $(n=40, p<0.05)^{26}$. They recommended that the surgical mask be changed after each individual procedure, especially those greater than two hours in duration ${ }^{26}$. This study was followed up by Liu et al. ${ }^{27}$ who investigated several factors that could affect surgical mask bioburden including type of surgical mask, conversation during surgery and face washing before applying a surgical mask. They identified that mask bioburden was higher when the wearer spoke ${ }^{27}$. Findings revealed that, in addition to wearing a surgical mask correctly as recommended by the national standards, surgical masks should be changed after each procedure, especially when usage extended over two hours, and unnecessary talking should be limited during procedures in order to minimise the risk of contamination of a surgical mask and to maximise optimal outcomes for both patients and theatre personnel ${ }^{26,27}$.

The practice of eliminating nonessential conversations in critical phases of surgery has also been shown to decrease distraction and enhance patient safety ${ }^{28}$.

\section{Implications for perioperative nursing practice and knowledge translation}

Perioperative nurses play an important role in delivering the best evidence-based outcomes for patients and fellow staff as well as coordinating the multidisciplinary team in operating theatres to provide evidence-based care. Perioperative nurses' responsibilities are to:

- remain up to date in their knowledge of the latest ACORN and national standards ${ }^{6}$ and ensure the local policies in the individual health care settings are up to date

- discuss the implications of a higher airborne biological load with the perioperative team ${ }^{13,14}$

- understand that face masks lower the biological load for bearded men $^{15}$

- realise that face masks should be worn correctly and changed every two hours and after each case $17,19,20$

- ensure all perioperative staff and students in the OR are always made aware of the possibility of blood spray reaching both scrubbed and non-scrubbed team members and the importance of wearing appropriate PPE $19,24,25$

- speak slowly and clearly when wearing a face mask because of the 
impact of face masks on speech intelligibility ${ }^{23}$

- be aware of the possible negative impact of wearing surgical masks during interaction with conscious patients - it may be beneficial to remove a surgical mask in the anaesthesic room or during induction to instil confidence and trust, but ensure that it is worn during surgery ${ }^{21,22}$.

\section{Conclusion and recommendations}

This integrated review shows that there is a statistically significant association between surgical face masks fitted over the face and nose and a lower biological load in air in the $\mathrm{OR}^{13-16}$ and that surgical face masks are a vital component of PPE.

For decades one of the reasons that perioperative nurses have worn face masks was to assist in the prevention of SSIs for patients. The causes of SSIs are multifactorial and to date no single study has taken into account all possible variables, thus reliable links between SSIs and wearing surgical face masks have not been made.

We do, however, know two important statistically significant facts:

1. wearing surgical face masks is a vital part of PPE, providing staff with protection from blood and body fluid splash 19,24,25

2. wearing a face mask decreases the biological load in the theatre environment $\mathrm{t}^{13,14}$.

While it is unknown what part the latter may play in SSIs, the decrease in CFU suggests a lowered risk of perioperative team members spreading airborne contaminants ${ }^{13,14}$. With the backdrop of a global pandemic, thinking may alter, increasing the significance of wearing surgical face masks to prevent the spread of respiratory infections between theatre staff who work in close proximity.

Limitations of this review include the fact that despite a thorough and systematic search being undertaken some papers may have been unintentionally missed. Papers from non-English speaking authors were excluded thus some international research may have also been omitted.

Findings from this integrated review would suggest two recommendations for future research. The first is that further research into the prevention of SSIs should investigate all variables, including patient comorbidities such as diabetes, and possible causative factors such as hypothermia and the wearing of face masks. The second is to consider future qualitative research into attitudes towards wearing PPE as attitudes may have changed due to new knowledge and thinking that has arisen from the COVID-19 pandemic.

In conclusion, wearing a surgical face mask has been shown to assist in preventing respiratory infections, by lowering the biological load in the theatre environment, and protecting staff from splash of blood and body fluids.

\section{Acknowledgment}

This paper was submitted to the University of Tasmania as part fulfilment of subject CNA803, Advanced Clinical Nursing Practice, for the Master of Clinical Nursing (Perioperative Nursing). The author sincerely wishes to thank Dr Paula Foran, unit coordinator, for her guidance throughout the master course and work in preparing this paper for publication.

\section{References}

1. Baldini A, Blevins K, Del Gaizo D, Enke O, Goswami K, Griffin W et al. General assembly, prevention, operating room - personnel: Proceedings of international consensus on orthopaedic infections. J Arthroplasty 2019;34(2S):S97S104. doi: 10.1016/j. arth.2018.09.059.

2. Birgand G, Saliou P, Lucet JC. Influence of staff behaviour on infections risk in operating rooms: What is the evidence? Infect Control Hosp Epidemiol 2015;36(1):93106. doi: $10.1017 /$ ice.20149.

3. Liu Z, Dumville JC, Norman G, Westby MJ, Blazeby J, McFarlane E et al. Intraoperative interventions for preventing surgical site infection: An overview of Cochrane reviews. Cochrane Database Syst Rev 2018;2(2):CD012653. doi: 10.1002/14651858. CD012653.pub2.

4. Da Zhou C, Sivathondan P, Handa A. Unmasking the surgeons: The evidence base behind the use of facemasks in surgery. J R Soc Med 2015;108(6):223-228. doi: $10.1177 / 0141076815583167$.

5. Vincent M, Edwards P. Disposable surgical face masks for preventing surgical wound infection in clean surgery. Cochrane Database Syst Rev 2016;4(4):CD002929. doi: 10.1002/14651858.CD002929.pub3.

6. Australian College of Perioperative Nurses Ltd (ACORN). Standards for Perioperative Nursing in Australia $16^{\text {th }}$ ed. Adelaide: ACORN; 2020.

7. Whittemore R, Knafl $K$. The integrative review: Updated methodology. J Adv Nurs 2005;52(5):546-553. doi: 10.1111/j.13652648.2005.03621.x

8. Lobionso-Wood G, Harber J. Nursing Research: Methods and Critical Appraisal for Evidence-based Practice $9^{\text {th }}$ ed. Elsevier: Sydney; 2018.

9. PRISMA. Preferred reporting items for systematic reviews and meta-analyses: The PRISMA statement [Internet]. Ottawa: PRISMA; 2009 [cited 2020 August 20]. Available from: www.prisma-statement.org/ PRISMAStatement/FlowDiagram.

10. Critical Appraisal Skills Programme. CASP Randomised controlled trial checklist 2018 [Internet]. Oxford: CASP; 2018 [updated 2020 May 1, cited 2020 August 20]. Available from: casp-uk.net/wp-content/uploads/2018/01/ CASP-Randomised-Controlled-TrialChecklist-2018.pdf.

11. Critical Appraisal Skills Programme. CASP cohort study checklist 2018. Oxford: CASP; 2018 [cited 2020 August 20]. Available from: casp-uk.net/wp-content/uploads/2018/01/ CASP-Cohort-Study-Checklist_2018.pdf. 
12. National Health and Medical Research Council (NHRMC). NHMRC levels of evidence and grades for recommendations for developers of guidelines. Canberra: NHRMC; 2009. Available from: www.nhmrc.gov.au/ sites/default/files/images/NHMRC\%20 Levels\%20and\%20Grades\%20(2009).pdf

13. Howard RA, Lathrop GW, Powell N. Sterile field contamination from powered air-purifying respirators (PAPRs) versus contamination from surgical masks. Am J Infect Control 2020;48(2):153-156. doi: doi org/10.1016/j.ajic.2019.08.009.

14. Stockwell RE, Wood ME, He C, Sherrard LJ, Ballard EL, Kidd et al. Face masks reduce the release of Pseudomonas aeruginosa cough aerosols when worn for clinically relevant periods. Am J Respir Crit Care Med 2018;198(10):1339-1342. doi: 10.1164/ rccm.201805-0823LE.

15. Parry JA, Karau MJ, Aho JM, Taunton M, Pate R. To beard or not to beard? Bacterial shedding among surgeons. Orthopedics 2016;39(2):e290-4. doi: 10.3928/0147744720160301-01.

16. Patel RB, Skaria SD, Mansour MM, Smaldone GC. Respiratory source control using a surgical mask: An in vitro study. J Occup Environ Hyg 2016;13(7):569-576. doi: 10.1080/15459624.2015.1043050.
17. Loison G, Troughton R, Raymond F, Lepelletier D, Lucet JC, Avril, C et al. Compliance with clothing regulations and traffic flow in the operating room: A multi-centre study of staff discipline during surgical procedures. J Hosp Infect 2017;96(3);281-285. doi: 10.1016/j. jhin.2017.03.026.

18. Dallolio L, Raggi A, Sanna T, Mazzetti M, Orsi A, Zanni A et al. Surveillance of environmental and procedural measures of infection control in the operating theatre setting. Int J Environ Res Public Health 2017;15(1). doi: 10.3390/ijerph15010046.

19. Herron JBT, Kuht JA, Hussain AZ, Gens KK, Gilliam AD. Do theatre staff use face masks in accordance with the manufacturers' guidelines of use? J Infect Prev 2019;20(2):99-106. doi: 10.1177/1757177418815551.

20. Kang J, O'Donnell JM, Colaianne B, Bircher $\mathrm{N}$, Ren D, Smith KJ. Use of personal protective equipment among health care personnel: Results of clinical observations and simulations. Am J Infect Control 2017;45(1):17-23. doi: 10.1016/j.ajic.2016.08.011.

21. Ahmad M, Humayun Mohmand M, Ahmad T. A survey among plastic surgeons wearing a mask in operating room. World J Plast Surg 2019;8(1):93-96. doi: 10.29252/wjps.8.1.93.

22. Wong CKM, Yip BHK, Mercer S, Griffiths S, Kung, K, Wong $M$ et al. Effect of facemasks on empathy and relational continuity: A randomised controlled trial in primary care. BMC Fam Pract 2013;14:200. Available from: www.biomedcentral.com/1471-2296/14/200.
23. Palmiero AJ, Symons D, Morgan JW $3^{\text {rd }}$, Shaffer RE. Speech intelligibility assessment of protective facemasks and air-purifying respirators. J Occup Environ Hyg 2016;13(12):960-968. doi: 10.1080/15459624.2016.1200723.

24. Cook CR, Gaston T, Woods B, Orozco F, Ong A, Radcliff K. Operative field debris often rises to the level of the surgeon's face shield during spine surgery: Are orthopedic space suits a reasonable solution? Int I Spine Surg 2019;13(6):501-6. doi: 10.14444/6067.

25. Spruce L. Surgical attire - a matter of preference or evidence? ORNAC journal 2016;34(1):14-35

26. Zhiqing L, Yongyun $C$, Wenxiang $C$, Mengning $Y$, Yuanqing $M$, Zhenan Z et al. Surgical masks as source of bacterial contamination during operative procedures. J Orthop Translat 2018;14:57-62. doi: 10.1016/j. jot.2018.06.002.

27. Liu Z, Yu D, Ge Y, Wang L, Zhang J, Li H et al. Understanding the factors involved in determining the bioburdens of surgical masks. Ann Transl Med 2019;7(23):754. doi: 10.21037/atm.2019.11.91.

28. Mackenzie S, Foran P. The impact of distractions and interruptions in the operating room on patient safety and the operating room team: An integrative review. JPN 2020;33(3):e-34-e-43. doi: 10.26550/22091092.1098 . 